\title{
Catalyst-Free Polymerization with 100\% Atom Economy: Facile Synthesis of Polysulfonates with Multifunctionalities
}

\author{
Xiaolin Liu, ${ }^{\dagger, \#}$ Xin Liang,,,$\#$ Yubing Hu, ${ }^{\dagger,}, \#$ Qing Qu, ${ }^{\dagger}$ Dongming Liu, $"$ Haotian Bai, ${ }^{\dagger}$ Ryan T. K.

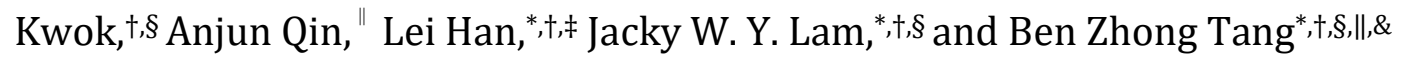 \\ ${ }^{\dagger}$ Department of Chemistry, Hong Kong Branch of Chinese National Engineering Research Center for Tissue Restoration \\ and Reconstruction, and Institute for Advanced Study, The Hong Kong University of Science and Technology, Clear \\ Water Bay, Kowloon, Hong Kong, China \\ * College of Chemistry and Pharmaceutical Sciences, Qingdao Agricultural University, 700 Changcheng Road, Qingdao, \\ Shandong, 266109 China \\ $\S$ HKUST-Shenzhen Research Institute, No. 9 Yuexing 1st RD, South Area, Hi-tech Park, Nanshan, Shenzhen 518057, \\ China \\ $\Phi$ Nano Science and Technology Program and William Mong Institute of Nano Science and Technology,The Hong Kong \\ University of Science and Technology, Clear Water Bay, Hong Kong, China \\ $\|$ Center for Aggregation-Induced Emission, SCUT-HKUST Joint Research Institute, State Key Laboratory of Luminescent \\ Materials and Devices, South China University of Technology, Guangzhou 510640, China \\ \& AIE Institute, Guangzhou Development District, Huangpu, Guangzhou 510530, China
}

\begin{abstract}
Polyesters are indispensable to many biological phenomena and the origins of life. For biomimicry and expansion of the family of polyesters, it is essential to develop facile strategies for their synthesis and investigate their properties profoundly. Compared with polyphosphate and polycarboxylate, polysulfonate was much less explored due to the synthetic difficulty. In this study, we developed catalyst-free spontaneous polymerizations of dihaloalkynes and disulfonic acids for the synthesis of polysulfonates at room temperature in air with $100 \%$ atom economy in high yields. The resulting halogen-rich polysulfonates were found to possess various properties, including post-functionalization, extraordinarily high refractive index, controllable visible photodegradation with strong photoacid generation, multi-color and 3D photopatterning with aggregation-induced emission, and practical broad-spectrum antibacterial activity.
\end{abstract}

\section{INTRODUCTION}

Many natural biomacromolecules possess polyester backbones $^{1-2}$, because they are indispensable to many important biological phenomena and play significant roles in the origins of life $^{3-4}$. For example, DNAs and RNAs are polyphosphate esters and plant cuticle is composed mainly of polycarboxylate esters. ${ }^{5}$ For biomimicry and also due to their excellent energy storage ability, biocompatibility and mechanical properties, man-made polyesters have been widely prepared. ${ }^{6-11}$. On the other hand, polyphosphate and polysulfonate esters both belong to heteroatom-containing polyesters. However, the latter ones have been scarcely explored due to their synthetic difficulty. Traditionally, polysulfonates are synthesized through interfacial polycondensations of aromatic sulfonyl chlorides and phenols. Unfortunately, this method is not suitable for multifarious monomers and the polymeric products obtained often show large polydispersity with low atom economy.12-13
In 2017, Sharpless et al. reported an efficient sulfur(VI) fluoride exchange (SuFEx) click reaction for the synthesis of polysulfonates in a short reaction time and a high yield with a high molecular weight, a narrow polydispersity and broad monomer scopes. ${ }^{14-16}$ Such a result is a big step forward and opens an avenue for the simple synthesis of polysulfonates ${ }^{17}$. Because such a reaction is still new, there is still a big room for improvement. For example, the SuFEx polymerization is required to be conducted under heating, dry condition and inert gas protection, and the structures of the monomers and bifluoride salt catalysts are rather unique and expensive. Additionally, the problem of atom economy is a concern because tert-butyldimethylsilyl fluoride is released as a byproduct. Thus, it is interesting and significant to establish a catalyst-free polymerization for preparing polysulfonates with $100 \%$ atom economy at room temperature in air using inexpensive monomers.

On the other hand, heteroatom-containing polymers have been found to show novel properties. For example, sulfurcontaining macromolecules such as polythiophene and 
polyphenylene sulfide have been proven to show extraordinary excellent mechanical properties, high stability, high refractive index ${ }^{18}$ and optoelectronic properties. ${ }^{19-20}$ Theoretically polysulfonates should also possess similar properties but such a speculation is not confirmed as their variety is still very limited. The previous research mainly focuses on their high thermal stability and utilizes them as commodity polymers or engineering plastics ${ }^{16}$. Actually, except the above-mentioned ones, polysulfonates are anticipated to possess unique functionalities. Considering that the sulfonate ester group is photo-responsive ${ }^{21-23}$, the polysulfonates could possibly undergo photodegradation readily with photoacid generation. Such a property may find promising applications in (bio-)pattern generation, antimicrobial and tissue engineering.

In this work, we developed a catalyst-free spontaneous polymerization from the organic reaction of commercially available inexpensive disulfonic acids and readily accessible dihaloalkynes $^{24}$ for the synthesis of polysulfonates in excellent yields and high molecular weights (Scheme 1). This catalyst-free polymerization was conducted at room temperature in air in a short reaction time with $100 \%$ atom economy. The resulting halogen-rich polysulfonates were demonstrated to possess multifunctionalities, including high refractive index, visible photodegradation with efficient photoacid generation, multi-color and 3D fluorescent photopatterning, broad-spectrum antibacterial activity and so on.

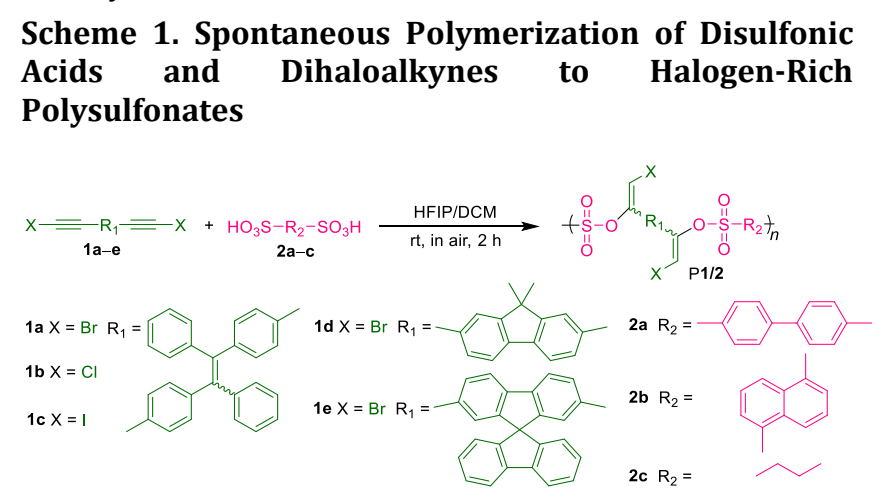

\section{RESULTS AND DISCUSSION}

Polymerization. Monomers used in this work are easily available. Disulfonic acids $\mathbf{2 a - 2 c}$ were purchased commercially, inexpensive and used without further purification. Dihaloalkynes 1a-1e were easily synthesized in high yields according to the previously reported procedures ${ }^{25-26}$. All the polymerizations were conducted under air at room temperature in a one-pot catalyst-free manner without generating any byproduct.

To obtain soluble polysulfonates in high yields with high molecular weights, we systematically investigated the polymerization conditions using $\mathbf{1 a}$ and $\mathbf{2 a}$ as monomers. The solvent, concentration and reaction time were carefully investigated (Table 1). The effect of solvent on the polymerization was first investigated (entries $1-3$, Table 1 ). Dichloromethane (DCM) is a good solvent for haloalkynes but is a poor solvent for sulfonic acids, while hexafluoro-2propanol (HFIP) is a good solvent for sulfonic acids because of the strong hydrogen bonding interaction (Figure S1 and S2 in supporting information) but is a poor solvent for haloalkynes. To achieve the best solving power for all monomers, HFIP/DCM mixtures with various volume fraction of HFIP and DCM were used for polymerization. As shown in Table 1 , the best polymerization result was obtained in HFIP/DCM (v/v, 1/8), which gave polymer with a weight-average molecular weight $\left(M_{\mathrm{w}}\right)$ of 13800 in $88 \%$ yield (entry 3). A polymer with a higher molecular weight was obtained in a solvent mixture with a lesser HFIP fraction probably because it is a poor solvent for the resulting polymer. In addition, the monomer concentration (entries 3-4, Table 1) also played a crucial role for the polymerization. When the monomer concentration of $\mathbf{1 a}$ was increased from $0.1 \mathrm{M}$ to $0.2 \mathrm{M}$, a polymer with a higher $M_{\mathrm{w}}$ was obtained in a higher yield (entry 4). However, further increasing the concentration of $1 \mathrm{a}$ to $0.4 \mathrm{M}$ led to a sharp decrease in both $M_{\mathrm{w}}$ and yield as the monomers were not completely dissolved. A time of $2 \mathrm{~h}$ was adopted as the optimal reaction time, since prolonging the reaction time to even $8 \mathrm{~h}$ did not lead to higher $M_{\mathrm{w}}$ and yield (entries 4-6).

Table 1. Optimization of the Model Polymerization Reaction $^{a}$

\begin{tabular}{ccccccc}
\hline entry & $\begin{array}{c}\text { HFIP: } \\
\text { DCM }\end{array}$ & $\begin{array}{c}{[\mathbf{1 a}]} \\
(\mathrm{M})\end{array}$ & $\begin{array}{c}\text { Time } \\
(\mathrm{h})\end{array}$ & $\begin{array}{c}\text { yield } \\
(\%)\end{array}$ & $M_{\mathrm{w}}{ }^{b}$ & $M_{\mathrm{w} /} M_{\mathrm{n}}{ }^{b}$ \\
\hline 1 & $1: 1$ & 0.10 & 2 & 90 & 5900 & 1.5 \\
2 & $1: 4$ & 0.10 & 2 & 84 & 10100 & 1.9 \\
3 & $1: 8$ & 0.10 & 2 & 88 & 13800 & 2.3 \\
$\mathbf{4}$ & $\mathbf{1 : 8}$ & $\mathbf{0 . 2 0}$ & $\mathbf{2}$ & $\mathbf{8 3}$ & $\mathbf{2 7 6 0 0}$ & $\mathbf{2 . 3}$ \\
5 & $1: 8$ & 0.20 & 4 & 83 & 29900 & 2.4 \\
6 & $1: 8$ & 0.20 & 8 & 84 & 27700 & 2.3 \\
\hline
\end{tabular}

${ }^{a}$ Unless otherwise noted, polymerizations were carried out at room temperature in air with $[\mathbf{1 a}]=[\mathbf{2 a}] .{ }^{b}$ Estimated by GPC in THF on the basis of a linear polystyrene calibration.

To reveal the kinetic of this polymerization, in-situ IR spectrscopy was used to monitor the formation of polysulfonates. The reaction progress of monomer $\mathbf{1 a}$ and 2a was monitored in air at room temperature. It is obvious that two new peaks corresponding to $\mathrm{C}-\mathrm{O}$ and $\mathrm{C}=\mathrm{C}$ stretching vibrations emerged at $\sim 1391$ and $1604 \mathrm{~cm}^{-1}$ in the spectra of the resulting poymer (Figure $1 \mathrm{~B}$ and $1 \mathrm{C}$ ). The peak intensity at $\sim 1391$ and $1604 \mathrm{~cm}^{-1}$ increased rapidly with time and reached saturation within $2 \mathrm{~h}$, suggesting the fast rate and high efficiency of this reaction. (Figure 1A)

Under the optimized polymerization conditions, different monomer combinations were employed to evaluate the robustness and universality of this polymerization route. As shown in Table 2, dihaloalkynes 1a-c with different halogen atoms all reacted efficiently with $\mathbf{2 a}$ to afford polysulfonates in excellent yields with high molecular weights (entries 1-3). The polymerizations also proceeded well for fluorene-substituted haloalkyne 1d and spirobifluorene-substitued haloalkyne 1e with large steric and 2a (entries 4-5). Regarding the monomer scope of sulfonic acids, we found that all reacted efficiently with 1a to generate polysulfonates with excellent $M_{\mathrm{w}}$ in high yields, 

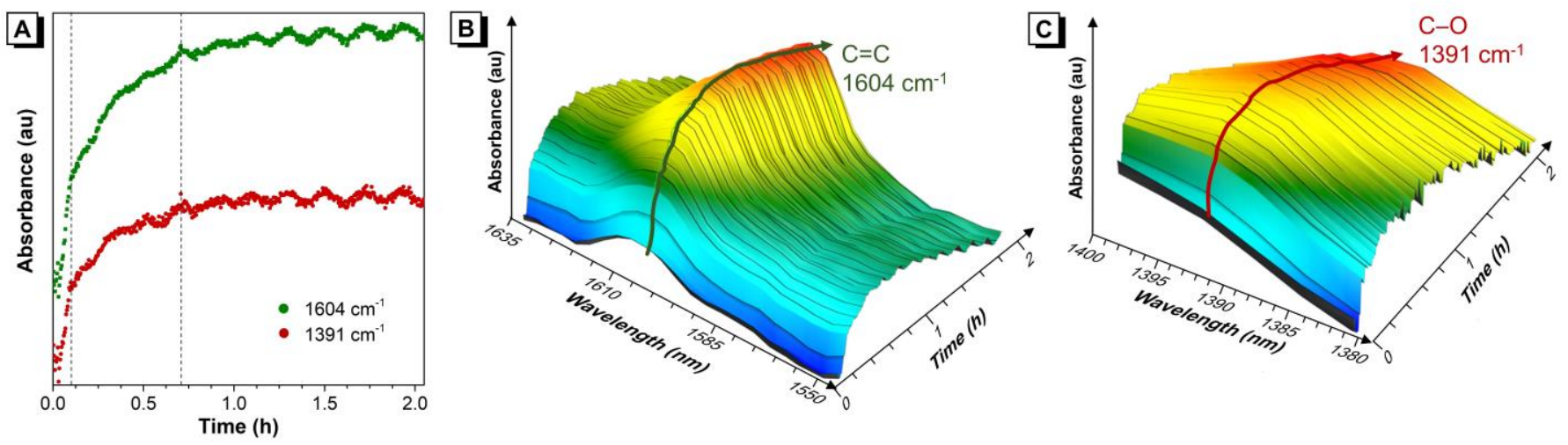

Figure 1. In-situ IR spectra of the polymerization conducted at room temperature for $2 \mathrm{~h}$ under the optimized conditions. (A) The time-dependent peak intensity at $1604 \mathrm{~cm}^{-1}$ and $1391 \mathrm{~cm}^{-1}$. Three-dimensional FT-IR profiles of the peaks at (B) $1604 \mathrm{~cm}^{-1}$ and (C) $1391 \mathrm{~cm}^{-1}$.

regardless their rigidity, aromaticity and bulkiness (entries 1, 6 and7). All the obtained polysulfonates showed excellent solubility in commonly used organic solvents, such as DMF, DMSO, THF, DCM and chloroform.

Table 2. Polymerization results of different monomers ${ }^{a}$

\begin{tabular}{ccccc}
\hline entry & monomer & yield (\%) & $M_{\mathrm{w}^{b}}$ & $M_{\mathrm{w} /} M_{\mathrm{n}}{ }^{b}$ \\
\hline 1 & $\mathbf{1 a} / \mathbf{2 a}$ & 83 & 27600 & 2.3 \\
2 & $\mathbf{1 b} / \mathbf{2 a}$ & 94 & 13900 & 1.5 \\
3 & $\mathbf{1 c} / \mathbf{2 a}$ & 91 & 12500 & 1.3 \\
4 & $\mathbf{1 d} / \mathbf{2 a}$ & 80 & 12800 & 1.7 \\
5 & $\mathbf{1 e} / \mathbf{2 a}$ & 88 & 11500 & 2.3 \\
$6^{c}$ & $\mathbf{1 a} / \mathbf{2 b}$ & 75 & 27000 & 2.8 \\
$7 c$ & $\mathbf{1 a} / \mathbf{2 c}$ & 72 & 16200 & 1.9 \\
\hline
\end{tabular}

aUnless otherwise noted, polymerizations were carried out at room temperature in air, with $[\mathbf{1 a}]=[\mathbf{2 a}]=0.20 \mathrm{M}$ in HFIP/DCM mixture (v/v, 1/8) for $2 \mathrm{~h} .{ }^{b}$ Estimated by GPC in THF on the basis of a linear polystyrene calibration. ${ }^{c}$ Reaction time was $4 \mathrm{~h}$.

Structural Characterization. To gain insight into the structures of the halogen-rich polysulfonates, model compound 1 was prepared by reaction of haloalkyne $\mathbf{1 a}$ and $p$-toluenesulfonic acid under the same synthetic conditions for the polymerization. Typical FT-IR, ${ }^{1} \mathrm{H}$ NMR and ${ }^{13} \mathrm{C}$ NMR spectra of polymer P1a/2a, model compound $\mathbf{1}$, and their corresponding monomers $\mathbf{1 a}$ and $\mathbf{2 a}$ were provided in Figure $\mathrm{S} 3$. The $\mathrm{C} \equiv \mathrm{C}$ stretching vibrations of $\mathbf{1 a}$ and $\mathrm{O}-\mathrm{H}$ stretching vibrations of 2 a occurred at 2194 and $3442 \mathrm{~cm}^{-1}$, respectively. These peaks were not observed in the spectra of 1 and P1a/2a. Meanwhile, new peaks associated with $\mathrm{C}=\mathrm{C}$ and $\mathrm{C}-\mathrm{O}$ appeared at 1604 and $1391 \mathrm{~cm}^{-1}$, indicating the successful occurrence of the polymerization. Similar observations were also found in the FT-IR spectra of other polymers (Figure S4 and S5).

The NMR spectra provided more detailed information about the polymer structures. The ${ }^{1} \mathrm{H}$ NMR spectrum of P1a/2a displayed a new peak emerged at $\delta 6.47$, corresponding to the vinyl proton at position "c" (Figure 2D). The ${ }^{13} \mathrm{C}$ NMR analysis further verified the polymer structure (Figure $2 \mathrm{E}-\mathrm{H}$ ). The characteristic peaks of the $\mathrm{C} \equiv \mathrm{C}$ resonances of $\mathbf{1 a}$ were not observed in the polymer spectrum. Instead, the carbon on position "c" resonated at $\delta$ 101.41. For all the 7 polysulfonates, the characteristic peaks of their vinyl protons absorbed at $\delta 6.30-6.75$, their ${ }^{13} \mathrm{C}$ NMR spectra showed no $\mathrm{C} \equiv \mathrm{C}$ carbon absorption peaks, confirming the successful synthesis of polymers with structures as shown in Scheme 1 (Figure S7-22).

Photophysical Properties. The absorption and emission spectra of dilute THF solutions $(40 \mu \mathrm{M})$ of the assynthesized polysulfonates were shown in Figure S25 and S26. The emission of polysulfonates is easily tunable from 440 to $550 \mathrm{~nm}$ by polymerization using monomers with different electron density (Figure S26).

By incorporating tetraphenylethylene into the polysulfonate skeleton, the resulting polymers $\mathbf{P} \mathbf{1 a}-\mathbf{c} / \mathbf{2 a}$, $\mathrm{P1a} / \mathbf{2 b}$ and P1a/2c showed aggregation-enhanced emission. Taking P1a/2a as an example, its DCM solution emitted weakly at $574 \mathrm{~nm}$. Upon addition of a non-polar poor solvent, such as hexane, the emission became stronger gradually, accompanied with a slight blue-shift in emission maximum to $558 \mathrm{~nm}$ (Figure S27 and S28).

Post-Functionalization. Post-functionalization is a powerful tool to endow polymers with more sophisticated structures and tunable functionalities. ${ }^{27-29}$ The vinyl bromine or vinyl iodine functionality of the halogen-rich polysulfonates enable them to undergo various postfunctionalization via efficient named organic reactions such as Suzuki coupling. To demonstrate such possibility, we conducted the reaction of $\mathrm{P} \mathbf{1 c} / \mathbf{2 a}$ with boronic acid derivative $3 \mathbf{a}$ in the presence of $\mathrm{Pd}\left(\mathrm{PPh}_{3}\right)_{4}$ and $\mathrm{K}_{2} \mathrm{CO}_{3}$ to generate P1c/2a/3a with an $M_{\mathrm{w}}$ of 9900 in a high yield of $79 \%$ and high conversion ratio of $88 \%$ (Scheme 2). This suggested that $\mathrm{P} \mathbf{1 c} / \mathbf{2 a}$ could serve as a versatile platform for post-functionalization to further enrich the variety and functionalities of the polysulfonates. After modification, the absorption maximum shifted from $340 \mathrm{~nm}$ to $350 \mathrm{~nm}$ (Figure S29), while the emission maximum moved from 530 $\mathrm{nm}$ to $550 \mathrm{~nm}$ (Figure S30) due to the extension of the conjugation. 


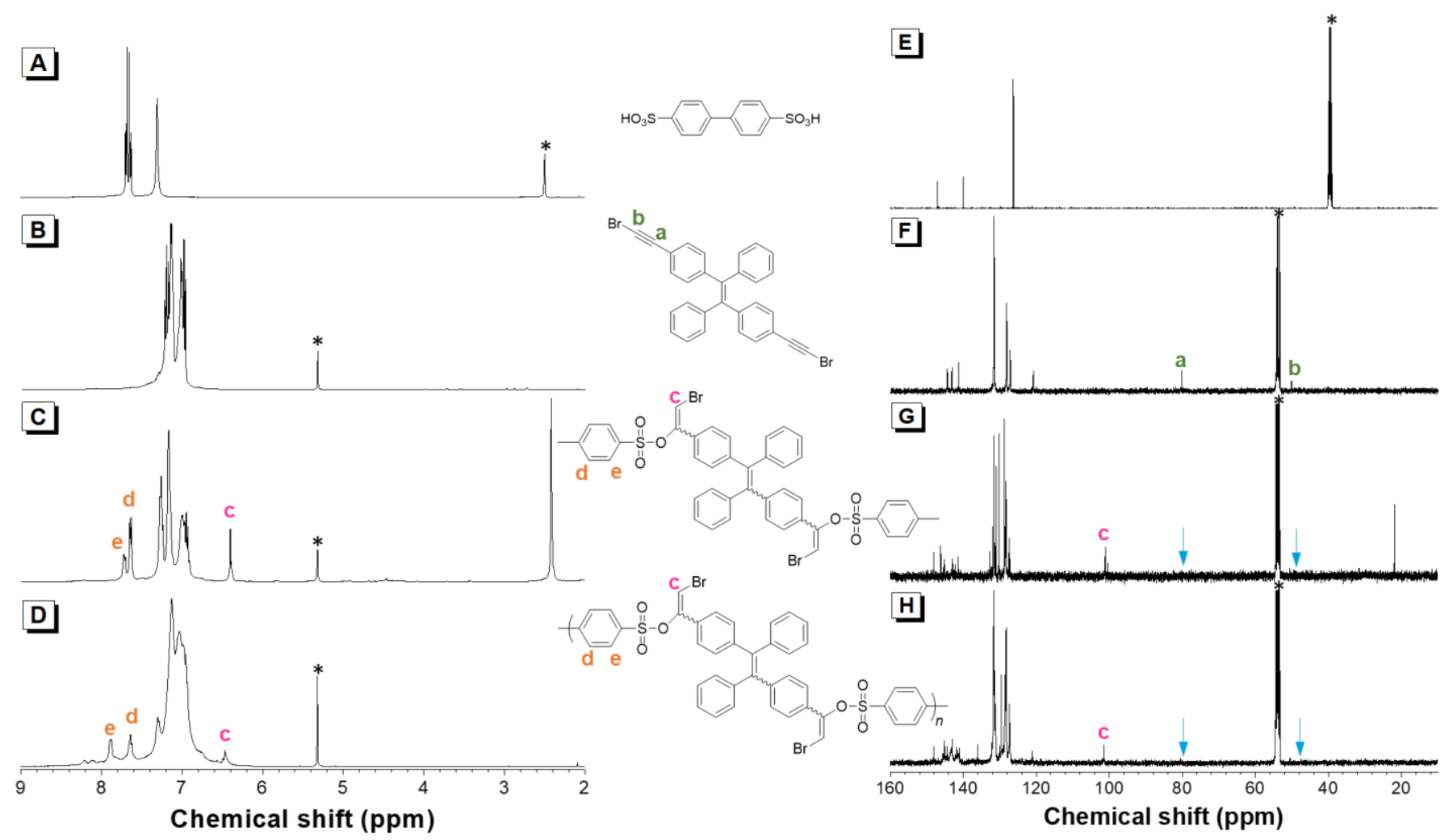

Figure 2. ${ }^{1} \mathrm{H}$ NMR spectra of (A) 2a in DMSO- $d_{6}$ and (B) 1a, (C) model compound 1 and (D) P1a/2a in $\mathrm{CD}_{2} \mathrm{Cl}_{2} .{ }^{13} \mathrm{C}$ NMR spectra of (E) 1a in DMSO- $d_{6}$ and (F) 1a, (G) model compound 1 and (H) P1a/2a in $\mathrm{CD}_{2} \mathrm{Cl}_{2}$.

Scheme 2. Postfunctionalization to Polymer P1c/2a/3a

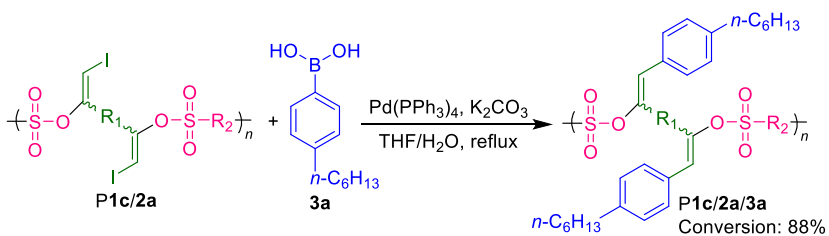

Photodegradation and Photoacid Generation. Some previous literatures have reported that aryl sulfonate decomposes upon exposure to UV irradiation. ${ }^{21,23,30}$ This inspired us to investigate whether the polysulfonates synthesized in this work could undergo readily photodegradation. As shown in Figure 3A, white light irradiation (400-780 nm) of a THF solution of P1a/2a with the intensity of $500 \mathrm{~mW} / \mathrm{cm}^{2}$ for 160 min exerted no change on the molecular weight of the polymer, suggestive of its super high stability. In contrast, when exposed the THF solution $(1 \mathrm{mg} / \mathrm{mL})$ of $\mathrm{P} \mathbf{1 a} / \mathbf{2 a}$ to $365 \mathrm{~nm}$ UV irradiation with the intensity of $40 \mathrm{~mW} / \mathrm{cm}^{2}$ for $160 \mathrm{~min}$, the $M_{\mathrm{n}}$ of the polymer gradually decreased from $12.1 \mathrm{kDa}$ to $1.9 \mathrm{kDa}$. The $M_{\mathrm{n}}$ of P1a/2a film also decreased to $4.3 \mathrm{kDa}$ under the same irradiation conditions. To our surprise, the photodegradation of the polymer could be accelerated in the presence of water. In a $\mathrm{H}_{2} \mathrm{O} / \mathrm{THF}$ mixture (v/v, 1/99), the $M_{\mathrm{n}}$ of P1a/2a decreased to $4.4 \mathrm{kDa}$ within only $5 \mathrm{~min}$ and the polymer was completely degraded in $60 \mathrm{~min}$. Dripping water on the $\mathbf{P} \mathbf{1 a} / \mathbf{2} \mathbf{a}$ film could also accelerate the photodegradation of the polymer.

The "on-water" photodegradation effect is possibly related to the formation of sulfonic acid. To study the acid generation during the photodegradation of $\mathrm{P} \mathbf{1 a} / \mathbf{2 a}, \mathrm{pH}$ assay was conducted in a $\mathrm{H}_{2} \mathrm{O} / \mathrm{THF}$ mixture $(\mathrm{v} / \mathrm{v}, 1 / 99)$ of P1a/2a (Figure $3 \mathrm{~B}$ ) using a pH meter for organic solvents equipped with $\mathrm{a} \mathrm{H}^{+}$ion sensitive glass electrode. The initial $\mathrm{pH}$ of the polymer solution was neutral and remained constant under strong white light irradiation $(400-780 \mathrm{~nm})$ with the intensity of $500 \mathrm{~mW} / \mathrm{cm}^{2}$. However, the $\mathrm{pH}$ of the polymer solution showed a sharp decrease upon prolonged $365 \mathrm{~nm}$ UV irradiation with the intensity of $40 \mathrm{~mW} / \mathrm{cm}^{2}$, and decreased from 7.1 to 0.3 within $20 \mathrm{~min}$, suggesting the formation of strong acid.

Interestingly, the photodegradation process could be visualized by naked eyes as the P1a/2a suspension in $\mathrm{THF} / \mathrm{H}_{2} \mathrm{O}$ mixture (v/v, 1/99) underwent a significant change of the emission maximum value from $495 \mathrm{~nm}$ to 427 nm during the photodegradation (Figure $3 \mathrm{C}$ and Table S2). The large fluorescence color change is mainly due to the decrease of conjugation length.

Based on the observed phenomenon, we proposed a mechanism of the photodegradation and photoacid generation (Scheme 3). The photodegradation was possibly initiated by a photoinduced homolytic S-O scission to generate a radical pair. Then the escaped sulfonyl radical 3 reacted with water to form the sulfonic acid product 4 . This demonstrated the on-water effect on the photodegradation. The phenoxyl radical 2 was probably transformed to vinyl alcohol 5, which underwent further tautomerization to generate the keto form $\mathbf{6}$. To testify the proposed mechanism, matrix-assisted laser desorption/ionizationtime of flight (MALDI-TOF) spectrum of the photodegradation product of model compound $\mathbf{1}$ was 

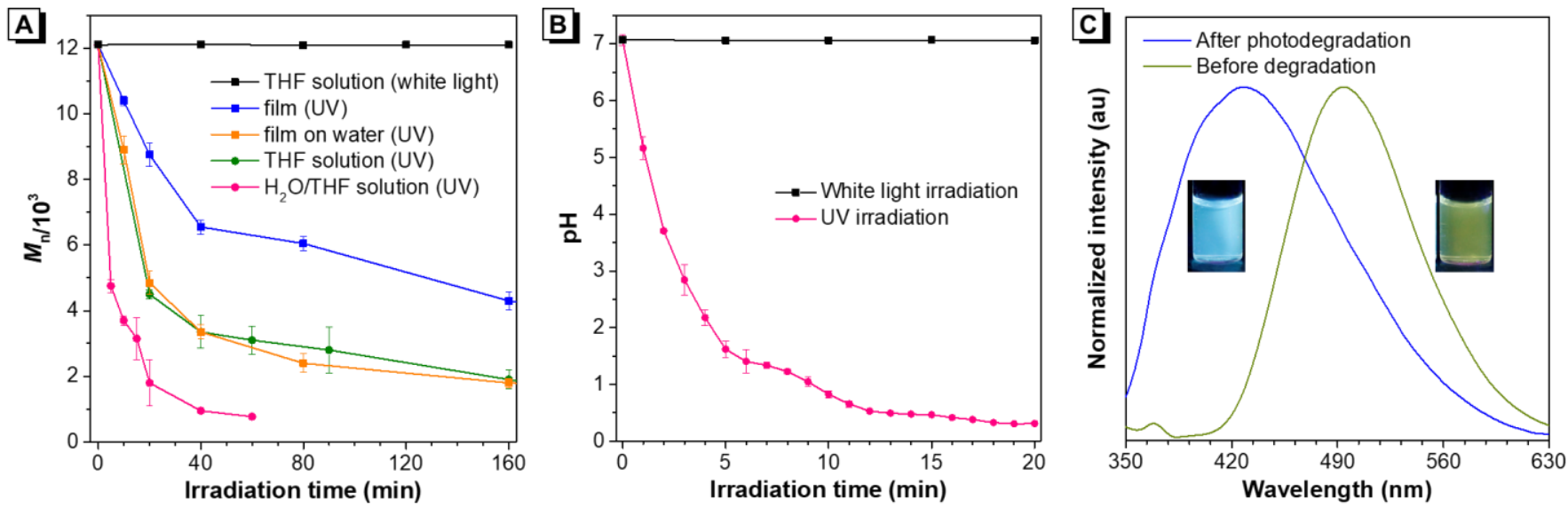

Figure 3. (A) Change of molecular weight of P1a/2a $(1 \mathrm{mg} / \mathrm{mL})$ in $\mathrm{THF}, \mathrm{H}_{2} \mathrm{O} / \mathrm{THF}$ mixture (v/v, 1/99), thin film or thin film with water at different irradiation time. (B) Change of $\mathrm{pH}$ of $\mathrm{P} 1 \mathrm{a} / \mathbf{2 a}(1 \mathrm{mg} / \mathrm{mL})$ in $\mathrm{H}_{2} \mathrm{O} / \mathrm{THF}$ mixture $(\mathrm{v} / \mathrm{v}, 1 / 99)$ at different irradiation time. (C) Emission spectra of $\mathrm{P} 1 \mathrm{a} / \mathbf{2 a}(100 \mu \mathrm{M})$ suspension in $\mathrm{THF} / \mathrm{H}_{2} \mathrm{O}$ mixture $(\mathrm{v} / \mathrm{v}, 1 / 99)$ before and after UV irradiation. Excitation wavelength: $330 \mathrm{~nm}$. Inset: corresponding fluorescent photographs of P1a/2a suspension taken under $365 \mathrm{~nm}$ UV irradiation.

measured, which demonstrated the formation of sulfonic acid product (Figure S32). In addition, the ${ }^{1} \mathrm{H}$ NMR spectra and ${ }^{13} \mathrm{C}$ NMR spectra of the degradation product also confirmed the disappearance of $\mathrm{C}=\mathrm{C}$ double bond and formation of keto and methylene groups (Figure S33 and S34).

\section{Scheme 3. Proposed Mechanism of Photoacid} Generation

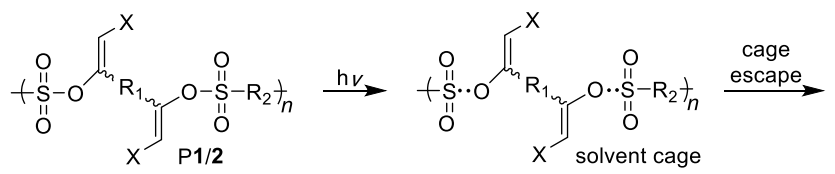

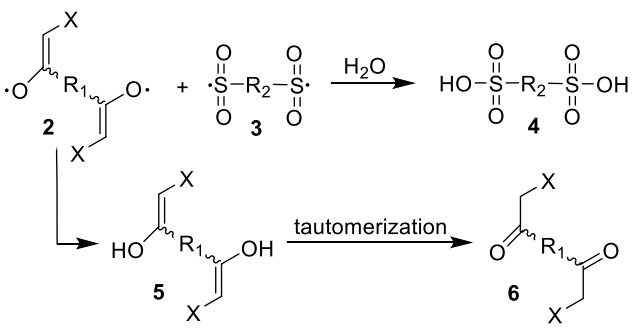

Light Refraction and Fluorescent Photopatterning. High-refractive-index polymers are promising candidate materials for optical engineering applications, such as optical chips, prisms, optical waveguides, memories and holographic image recording systems. ${ }^{11,31-33}$ Heteroatoms especially halogen elements and sulfur are well-known contributors for enhancing the refractive index $(n)$. Thus, being halogen and sulfur-containing polymers, we are interested to investigate the $n$ values of the synthesized polysulfonates.

The $n$ values of the polysulfonates were tunable by internal control. By incorporating different monomers into the polymer backbone, the polysulfonates P1a-d/2a showed high refractive indices ranging from 1.920 to 1.626 in the spectral range of 400-900 $\mathrm{nm}$ (Figure 4A). It is worth to note that the $n$ values of P1a/2a were 1.823 at $486.1 \mathrm{~nm}$ and 1.770 at $632.8 \mathrm{~nm}$, which were higher than those of sapphire (1.776 at $486.1 \mathrm{~nm}$ and 1.766 at $632.8 \mathrm{~nm})$. All the polysulfonates showed much higher $n$ values than those of the commercially important optical polymers, such as polycarbonate $\left(n_{486.1}=1.600\right.$ and $\left.n_{632.8}=1.581\right)$ and poly(methyl methacrylate $)\left(n_{486.1}=1.497\right.$ and $n_{632.8}=$ 1.489)..$^{34}$
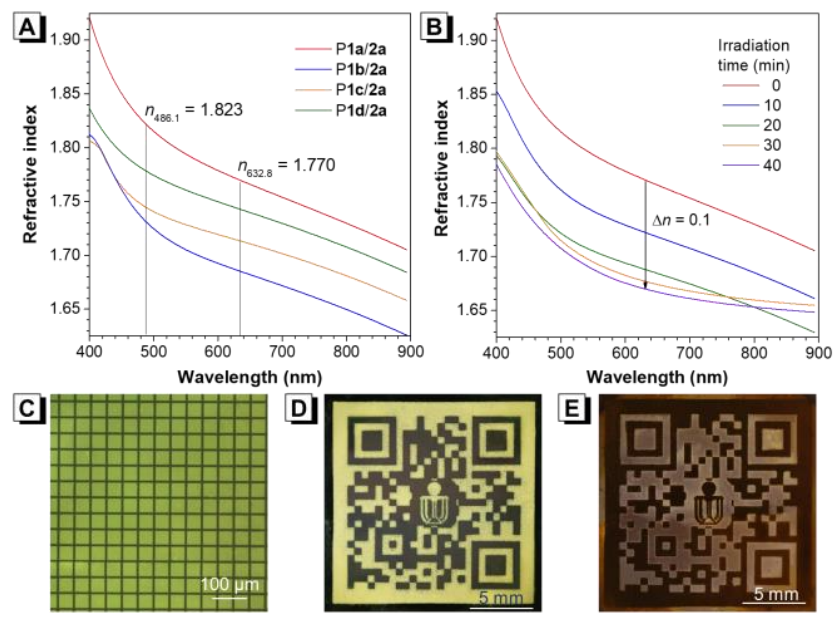

Figure 4. (A) Wavelength-dependent refractive index of thin films of $P 1 / 2$. (B) Wavelength-dependent of refractive index of thin films of P1a/2a at different UV irradiation time. (C and D) Two-dimensional fluorescent photopatterns generated by photolithography of films of P1a/2a. Excitation wavelength: $365 \mathrm{~nm}$. (E) Photographs of the photopatterns taken under normal room light.

Their $n$ values were also tunable by external control as the polysulfonates were photo-responsive. As shown in Figure $4 \mathrm{~B}$, the $n$ values of $\mathbf{P} \mathbf{1 a} / \mathbf{2 a}$ decreased gradually with prolonging the irradiation time and the $n$ difference $(\Delta n)$ at $632.8 \mathrm{~nm}$ before and after light irradiation by Mercury Arc Lamp (200-400 nm) for 40 min could be as large as 0.1 . The excellent tunability of the film refractivity by UV irradiation enables these polymers to find promising applications in optical data storage devices, gradient-index optics and integrated photonic technology. ${ }^{35}$ 
The generation of complex fluorescent patterns by photolithography technique is of great significance in terms of the optical writing and reading, anti-counterfeiting applications, and biological sensing systems. ${ }^{36}$ Because of the excellent solubility and film-forming ability of the present polysulfonates, their uniform films without defect can be easily fabricated on silica wafers by a simple spincoating technique. The photo-responsive behavior of the polysulfonates makes them excellent candidates for pattern generation. As shown in Figure 4C, under light irradiation by Mercury Arc Lamp (200-400 nm) in air for $40 \mathrm{~min}$ through a copper photomask, the polymer first underwent photodegradation, and subsequent photo-oxidation. Fluorescence of the exposed regions (lines) was completely quenched due to the photo-oxidation of the chromophores, while the emissive squares were protected by copper and remained intact. Thus, two-dimentional fluorescent patterns with high resolution and sharp edges were generated. By changing the shape and scale of the photomask, various patterns in different scales could be generated, such as QR code (Figure 4D). Surprisingly, these patterns could also be clearly observed under room light (Figure 4E), which was presumably due to the remarkable refractive index change and absorption change of the film after UV irradiation.

Multi-Color Fluorescent Photopatterning. The creation of highly ordered multi-color fluorescent patterns is meaningful but challenging. Previously reported methods are either time- consuming 37 or require mixtures of different

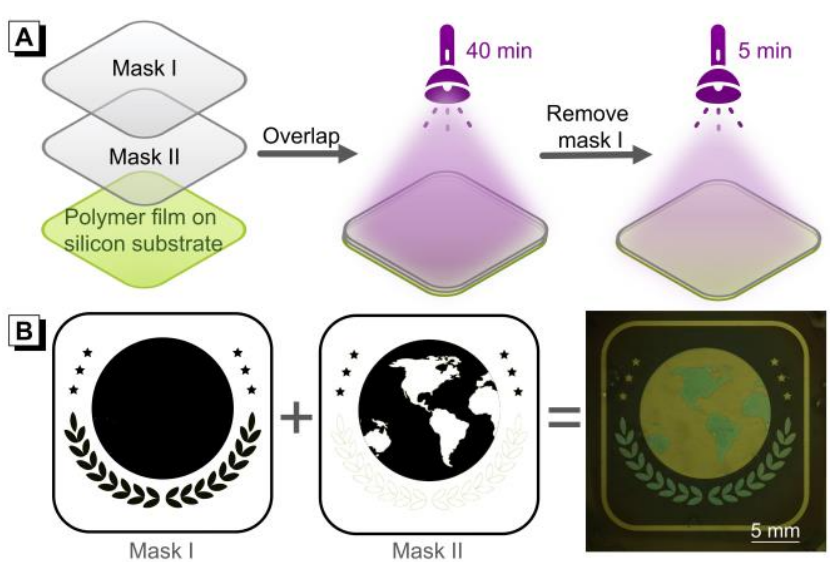

Figure 5. (A) Process of multi-color photopattern generation. (B) Images of the photo masks and photograph of the multicolor photopattern under UV irradiation at $365 \mathrm{~nm}$.

fluorescent materials ${ }^{38-39}$. However, using only a single material for multi-color luminescent photopattern is still rare. Considering that the fluorescence of $\mathrm{P} \mathbf{1 a} / \mathbf{2 a}$ blueshifted under UV irradiation for a short time due to photodegradation, and photobleached under UV irradiation for long time, we tried to fabricate multi-color fluorescent pattern using P1a/2a film. The polymer film was first irradiated by Mercury Arc Lamp (200-400 nm) through both photo mask I and II for $40 \mathrm{~min}$ to induced photobleaching. Then mask I was removed and the film was

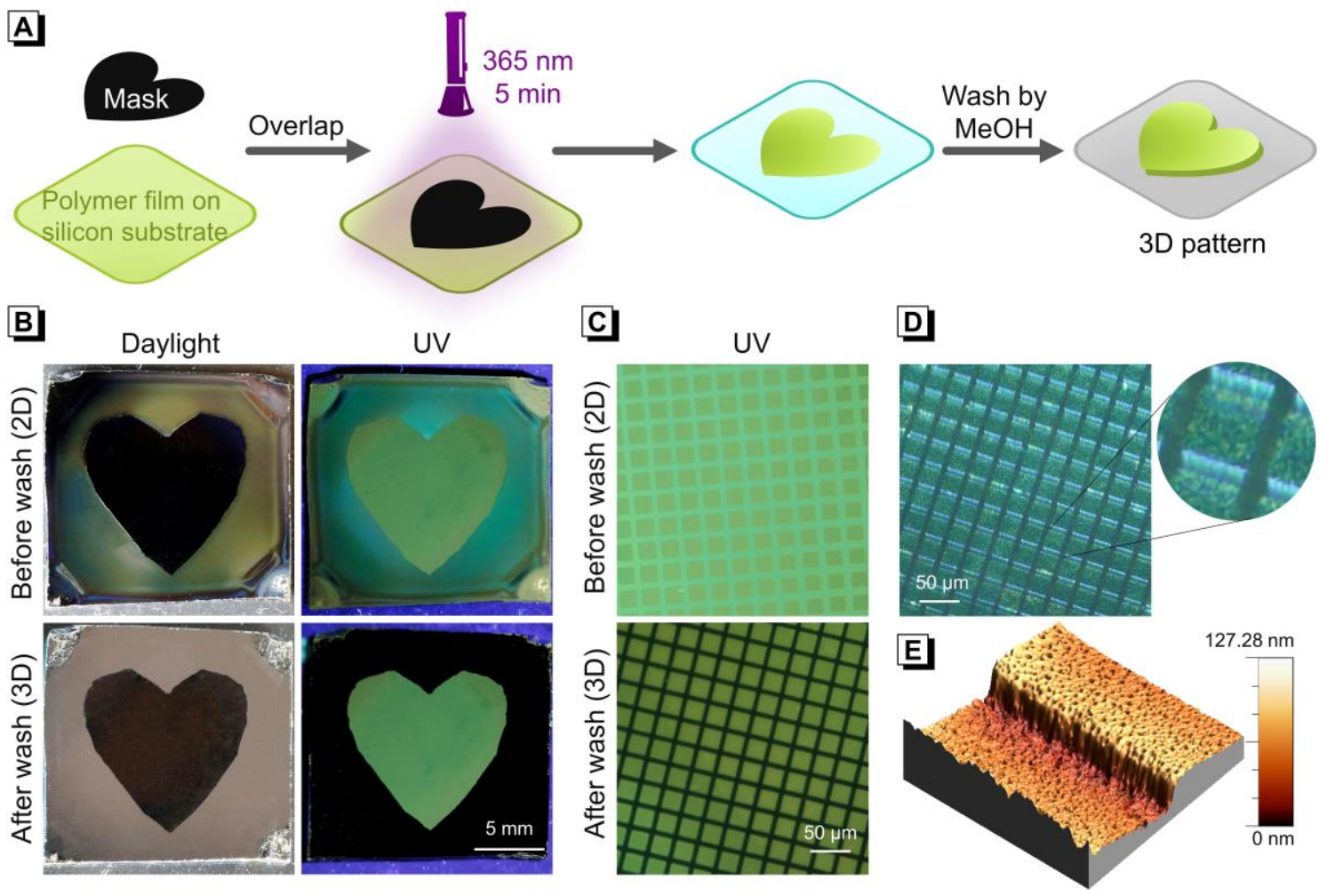

Figure 6. (A) Scheme of 3D photopattern generation. (B) Photographs of the 2D (upper) and 3D (bottom) heart-shape photopatterns taken under normal room light (left) or UV irradiation (right). Excitation wavelength: 330-385 nm. (C) Microscopic photographs of the 2D (upper) and 3D (bottom) grid-like photopatterns taken under $365 \mathrm{~nm}$ UV irradiation. (D) Photograph of the 3D grid-like sample with tilt angle observed under the bright-field of a microscope. (E) 3D AFM image of the 3D grid-like sample in a $10 \mu \mathrm{m}$ square. 

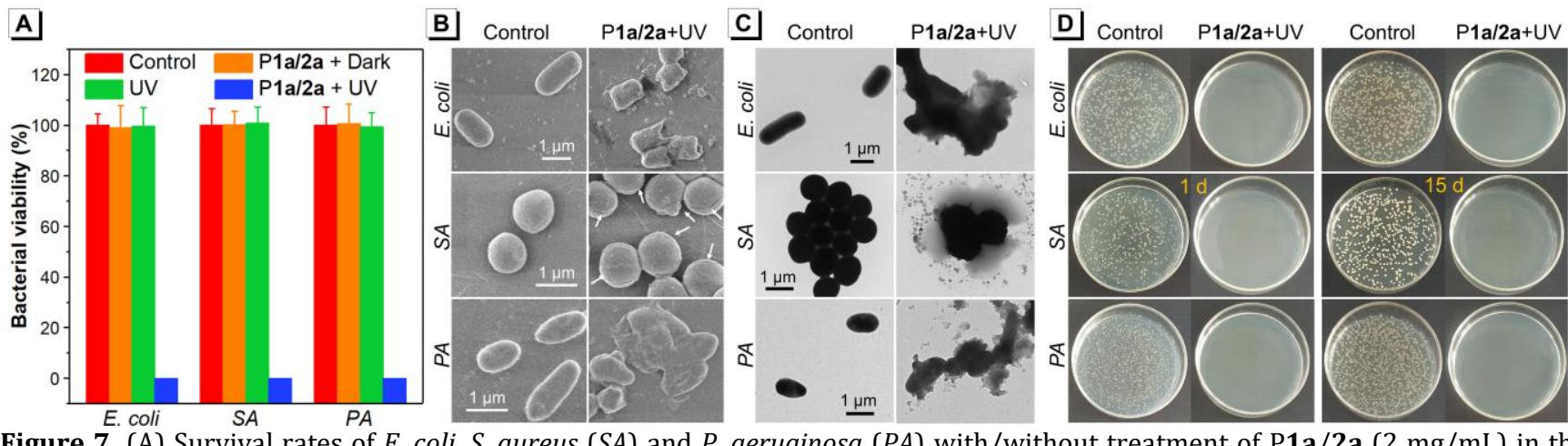

Figure 7. (A) Survival rates of E. coli, S. aureus $(S A)$ and $P$. aeruginosa $(P A)$ with/without treatment of $\mathrm{P} \mathbf{1 a} / \mathbf{2 a}(2 \mathrm{mg} / \mathrm{mL})$ in the presence or absence of light irradiation at $365 \mathrm{~nm}\left(40 \mathrm{~mW} \mathrm{~cm}^{-2}\right)$. (B) Morphology change of bacteria upon diverse treatments observed by SEM. (C) Morphology change of bacteria upon diverse treatments observed by TEM. (D) Photographs of agar plates of E. coli, $S A$ and $P A$ after 1 day and 15 days.

further irradiated for $5 \mathrm{~min}$ through mask II to trigger photodegradation (Figure 5A). As a result, a fluorescent "earth" picture with yellow, blue, and black color was finally generated (Figure 5B).

Three-dimensional Fluorescent Photopattern Generation. Natural creatures are experts and beneficiaries in fabricating micro- or nano-threedimensional (3D) ordered structures, such as the superhydrophobic surface of the lotus leaf and the structural coloration of the insects and animals. ${ }^{40-42}$ Thus, 3D ordered patterns have attracted considerable research interest due to their special functionalities. ${ }^{43}$ As the polysulfonates are highly sensitive to UV irradiation, they are ideal materials for 3D fluorescent pattern fabrication (Figure 6A). As shown in Figure 6B, after the polymer film was irradiated with $365 \mathrm{~nm}$ for $5 \mathrm{~min}$ through a heart-shape mask, the irradiated part showed blue-shifted fluorescence and remarkable change in refractive index due to photodegradation. By dipping the film in methanol for a few seconds, the irradiated part was wash away completely because the irradiated polymers lost their mechanical property. The protected part, on the contrary, was intact. Finally, a 3D heart-shape pattern was generated. By changing the scale and pattern of the photomask, a 3D fluorescent micro-array could be fabricated in the same way (Figure 6C). The 3D structure could be observed by slightly slanting the micro-array under the bright-field of the microscope (Figure 6D). The height of the step was measured to be $75 \mathrm{~nm}$ by atomic force microscopy (AFM) (Figure 6E). The facile, rapid and precisely controllable fabrication of fluorescent 3D ordered structure demonstrated the promising future of polysulfonates in constructing biomimetic materials, functional interfaces, and cost-efficient photolithography materials.

Controllable broad-spectrum bacteria killing. Controllable broad-spectrum bactericides and antibiotics are invaluable in the modern healthcare-associated infections. ${ }^{44-48}$ The ability of generating strong photoacid of the polysulfonates motivates us to further explore their antibacterial performance as $\mathrm{pH}$ value has significant influence on the bacterial growth. ${ }^{49}$
In a preliminary experiment, the UV-triggered $\mathrm{pH}$ change of bacteria resuspension containing $\mathrm{P} \mathbf{1 a} / \mathbf{2 a}$ was investigated and the $\mathrm{pH}$ was found to decrease from 6.7 to 2.3 after $365 \mathrm{~nm}$ UV irradiation for $30 \mathrm{~min}$ (Figure S35). It's worth noting that $\mathrm{pH}$ change of $\mathrm{P} \mathbf{1 a} / \mathbf{2 a}$ in bacteria resuspension was slower than that in $\mathrm{H}_{2} \mathrm{O} / \mathrm{THF}$ mixed solvent (Figure 3B), presumably due to the buffer capacity of the bacterial cytosol. Excitingly, under natural light, P1a/2a showed no toxicity to all the three bacteria even at a concentration of $2 \mathrm{mg} / \mathrm{mL}$ (Figure S36), which facilitated the controllable bacteria killing in ordinary circumstance. According to the preliminary experiments, $2 \mathrm{mg} / \mathrm{mL}$ $\mathrm{P} 1 \mathrm{a} / 2$ a solution and $30 \mathrm{~min}$ UV irradiation were adopted as experimental conditions in the subsequent experiments.

By virtue of the photoacid generation ability and hypotoxicity of $\mathrm{P} \mathbf{1 a} / \mathbf{2 a}$, the antimicrobial activities of P1a/2a were subsequently evaluated to representative pathogenic bacteria (Gram-positive $S$. aureus and Gramnegative $E$. coli and $P$. aeruginosa) by a traditional plate counting method. As shown in Figure 7A and Figure S37, in the absence of $\mathbf{P} \mathbf{1 a} / \mathbf{2 a}$, all three kinds of bacteria grew and reproduced smoothly on the agar plates in dark or UV irradiation. The dark toxicity of P1a/2a was inconspicuous on all bacteria. In the presence of both $\mathrm{P} \mathbf{1 a} / \mathbf{2 a}$ and UV irradiation, all three bacteria were killed effectively and no colony formation could be observed on the plates, implying the excellent broad-spectrum antibacterial effect of P1a/2a.

To further confirm the bacteria killing effect of P1a/2a, we employed scanning electron microscopy (SEM) and transmission electron microscopy (TEM) to visualize the morphological changes of bacterial cells under P1a/2a treatment selecting $S$. aureus, $E$. coli and $P$. aeruginosa as representatives (Figure 7B and 7C, Figure S38 and S39). Without P1a/2a or UV irradiation, all kinds of bacteria showed regular shape with well-defined borders and cell walls. When $E$. coli and P. aeruginosa were treated with P1a/2a and UV irradiation, obvious cell deformation, broken cell with the leakage of intracellular contents, and cell fragments were observed. $S$. aureus treated by P1a/2a and UV irradiation on the other hand, showed rough surface and fissured cell wall. 
Furthermore, the bacteria treated by P1a/2a and UV irradiation did not grow any more on agar plate after incubation at $37{ }^{\circ} \mathrm{C}$ for 15 days, (Figure 7D), which demonstrated the $100 \%$ irreversible bacteria killing effect of P1a/2a.
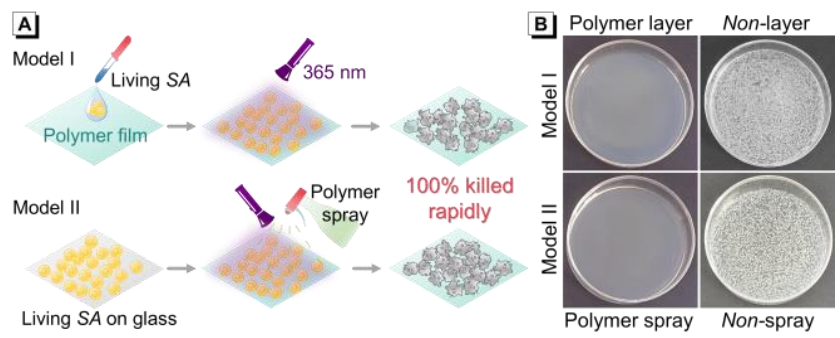

Figure 8. (A) Two antimicrobial methods for practical applications. (B) Photographs of the agar plates from two antimicrobial methods.

The above data indicated that the as-synthesized polymers at high concentration showed not only low dark toxicity but also low toxicity under visible light. Such a behavior differs from other aggregation-induced emission antibacterial agents ${ }^{50}$. Moreover, the as-synthesized polymers could cause high-efficient irreversible destruction of various pathogenic bacteria under UV irradiation. These characteristics will benefit the application of controllable in vitro sterilization in ordinary circumstance. Hence, for proof of concept, two practical sterilization models were designed by using P1a/2a as antibacterial coating or spray (Figure $8 \mathrm{~A}$ ). In model I, the polymer film was first coated on the glass and $S$. aureus was then transplanted on the film. After $365 \mathrm{~nm}$ UV irradiation for $30 \mathrm{~min}$, the bacteria were $100 \%$ killed under wet condition where bacteria could germinate (Figure 8B and Figure S40). In model II, the polymer suspension was sprayed on the glass slide covering with bacteria followed by the $365 \mathrm{~nm}$ UV irradiation for $30 \mathrm{~min}$. After plate cultivation, the bacteria were also found to be 100\% killed Figure 8B). These two models demonstrated that the polysulfonates are promising and flexible antibacterial materials to different situations, such as the surface sterilization of commodity, the resistance to corrosion caused by microorganisms in ship and the customized bacterial scaffold.

\section{CONCLUSION}

In summary, we developed a catalyst-free spontaneous polymerization for the synthesis of halogen-rich polysulfonates in $100 \%$ atom economy. The polymerization proceeded efficiently at room temperature in air within $2 \mathrm{~h}$, generating a series of main-chain polysulfonates in excellent yields (up to 94\%) and high molecular weights (up to 27 600) with wide monomer scope and excellent solubility. The halogen atoms of the polymers enable them to undergo various efficient postfunctionalization such as Suzuki coupling to further enrich the family of polysulfonates and endow them with advanced functionalities. These polysulfonates showed extraordinarily high and tunable refractive indices because of the halogen atoms and the sulfonate ester group. Notably, these polysulfonates showed controllable photodegradation with remarkable fluorescence change. Such a property allows the fabrication of 3D fluorescence photopattern and multi-color photopattern with only a single optical material. As strong photoacid generators, the polysulfonates could not only realize the UV-triggered, irreversible and broad-spectrum bacteria killing, but also showed hypotoxicity in dark or natural light. P1a/2a was also demonstrated to be a high-efficient bactericide as antibacterial layer or spray in two practical models. The catalyst-free spontaneous polymerization will provide new strategy for the facile synthesis of polysulfonates to greatly enrich the family of polyesters. The profound investigation on the properties and the various applications of polysulfonates will also provide more prospects for their practical usages, such as irreversible bacteria killing, tissue engineering, surface modification, 3D-structured interface, and bio-pattern fabrication.

\section{ASSOCIATED CONTENT}

\section{Supporting Information.}

This material is available free of charge via the Internet at http://pubs.acs.org.

Details of the materials, methods, synthetic procedures, and characterization data (IR, NMR, HRMS, etc.); photophysical properties and light refraction data of the polymers; photo of agar plates, SEM, and TEM images. (PDF)

\section{AUTHOR INFORMATION}

\section{Corresponding Author \\ * hanlei@qau.edu.cn \\ * chjacky@ust.hk \\ * tangbenz@ust.hk}

ORCID

Xiaolin Liu: 0000-0002-6909-117X

Lei Han: 0000-0003-1955-0718

Jacky W. Y. Lam: 0000-0001-5754-1703

Ben Zhong Tang: 0000-0002-0293-964X

\section{Author Contributions}

${ }^{\#}$ X.L., X.L. and Y.H. contributed equally to this work. Notes

The authors declare no competing financial interest.

\section{ACKNOWLEDGMENT}

This work was financially supported by the National Science Foundation of China (21788102 and 21705087), the Research Grants Council of Hong Kong (16304819, 16305618 and C6009-17G), the Innovation and Technology Commission (ITCCNERC14SC01), the National Key Research and Development program of China (2018YFE0190200), the Science and Technology Plan of Shenzhen (JCYJ20170818113602462, JCYJ20180306174910791, JCYJ20170818113530705 and JCYJ20180306180231853) and A Project of Shandong Province Higher Educational Science and Technology Program (J18KA067). X. L. L. thanks Prof. Zhiyang Liu from Southeast University and Prof. Hanchu Huang from Sun Yat-sen University for kind discussions and suggestions. X. L. L. also thanks Biosciences Central Research Facility of HKUST for technical assistance. 


\section{REFERENCES}

(1) Stang, P. J., Alkynyl Carboxylate, Phosphate, and Sulfonate Esters. Acc. Chem. Res. 1991, 24 (10), 304-310.

(2) Spring, F. S., Naturally Occurring Polyesters. Nature 1945, 155 (3931), 272-272.

(3) Jia, T. Z.; Chandru, K.; Hongo, Y.; Afrin, R.; Usui, T.; Myojo, K.; Cleaves, H. J., Membraneless Polyester Microdroplets as Primordial Compartments at the Origins of Life. Proc. Natl. Acad. Sci. U. S. A. 2019, 116 (32), 15830-15835.

(4) Chandru, K.; Guttenberg, N.; Giri, C.; Hongo, Y.; Butch, C.; Mamajanov, I.; Cleaves, H. J., Simple Prebiotic Synthesis of High Diversity Dynamic Combinatorial Polyester Libraries. Commun. Chem. 2018, 1 (1), 30.

(5) Kolattukudy, P. E., Biopolyester Membranes of Plants: Cutin and Suberin. Science 1980, 208 (4447), 990-1000.

(6) Tsao, Y.Y. T.; Smith, T. H.; Wooley, K. L., Regioisomeric Preference in Ring-Opening Polymerization of $3^{\prime}, 5^{\prime}$-Cyclic Phosphoesters of Functional Thymidine DNA Analogues. ACS Macro Lett. 2018, 7 (2), 153-158.

(7) Rashchi, F.; Finch, J. A., Polyphosphates: A Review Their Chemistry and Application with Particular Reference to Mineral Processing. Miner. Eng. 2000, 13 (10), 1019-1035.

(8) Samarajeewa, S.; Shrestha, R.; Li, Y.; Wooley, K. L., Degradability of Poly(Lactic Acid)-Containing Nanoparticles: Enzymatic Access through a Cross-Linked Shell Barrier. J. Am. Chem. Soc. 2012, 134 (2), 1235-1242. (9) Van Horn, B. A.; Iha, R. K.; Wooley, K. L., Sequential and Single-Step, One-Pot Strategies for the Transformation of Hydrolytically Degradable Polyesters into Multifunctional Systems. Macromolecules 2008, 41 (5), $1618-1626$

(10) Song, Y.; Ji, X.; Dong, M.; Li, R.; Lin, Y.-N.; Wang, H.; Wooley, K. L., Advancing the Development of Highly-Functionalizable Glucose-Based Polycarbonates by Tuning of the Glass Transition Temperature. J. Am. Chem. Soc. 2018, 140 (47), 16053-16057.

(11) Geervliet, T. A.; Gavrila, I.; Iasilli, G.; Picchioni, F.; Pucci, A., Luminescent Solar Concentrators Based on Renewable Polyester Matrices. Chemistry - An Asian Journal 2019, 14 (6), 877-883.

(12) Thomson, D. W.; Ehlers, G. F. L., Aromatic Polysulfonates: Preparation and Properties. J. Polym. Sci., Part A: Gen. Pap. 1964, 2 (3), 1051-1056.

(13) Campbell, R. W.; Hill, H. W., Polymerization of 4Hydroxybenzenesulfonyl Chloride. Macromolecules 1973, 6 (4), $492-$ 495.

(14) Wang, H.; Zhou, F.; Ren, G.; Zheng, Q.; Chen, H.; Gao, B.; Klivansky, L.; Liu, Y.; Wu, B.; Xu, Q.; Lu, J.; Sharpless, K. B.; Wu, P., Sufex-Based Polysulfonate Formation from Ethenesulfonyl Fluoride-Amine Adducts. Angew. Chem., Int. Ed. 2017, 56 (37), 11203-11208.

(15) Gao, B.; Zhang, L.; Zheng, Q.; Zhou, F.; Klivansky, L. M.; Lu, J.; Liu, Y.; Dong, J.; Wu, P.; Sharpless, K. B., Bifluoride-Catalysed Sulfur(Vi) Fluoride Exchange Reaction for the Synthesis of Polysulfates and Polysulfonates. Nat. Chem. 2017, 9 (11), 1083-1088.

(16) Xu, L.; Wu, P.; Dong, J., Chapter 1 New Polymers from Sufex Click Chemistry: Syntheses and Perspectives. In Synthetic Polymer Chemistry: Innovations and Outlook, The Royal Society of Chemistry: 2019; pp 131.

(17) Yang, C.; Flynn, J. P.; Niu, J., Facile Synthesis of Sequence-Regulated Synthetic Polymers Using Orthogonal Sufex and Cuaac Click Reactions. Angew. Chem., Int. Ed. 2018, 57 (49), 16194-16199.

(18) Higashihara, T.; Ueda, M., Recent Progress in High Refractive Index Polymers. Macromolecules 2015, 48 (7), 1915-1929.

(19) Kaloni, T. P.; Giesbrecht, P. K.; Schreckenbach, G.; Freund, M. S., Polythiophene: From Fundamental Perspectives to Applications. Chem. Mater. 2017, 29 (24), 10248-10283.

(20) Zuo, P.; Tcharkhtchi, A.; Shirinbayan, M.; Fitoussi, J.; Bakir, F., Overall Investigation of Poly (Phenylene Sulfide) from Synthesis and Process to Applications-a Review. Macromol. Mater. Eng. 2019, 304 (5), 1800686.

(21) Ariyasu, S.; Hanaya, K.; Tsunoda, M.; Kitamura, M.; Hayase, M.; Abe, R.; Aoki, S., Photochemical Cleavage Reaction of 8-Quinolinyl Sulfonates That Are Halogenated and Nitrated at the 7-Position. Chem. Pharm. Bull. 2011, 59 (11), 1355-1362.

(22) Xu, W.; Li, T.; Li, G.; Wu, Y.; Miyashita, T., Novel Polymeric Nonionic Photoacid Generators and Corresponding Polymer Langmuir-Blodgett (Lb) Films for Photopatterning. J. Photochem. Photobiol., A 2011, 219 (1), 50-57.
(23) Kageyama, Y.; Ohshima, R.; Sakurama, K.; Fujiwara, Y.; Tanimoto, Y.; Yamada, Y.; Aoki, S., Photochemical Cleavage Reactions of 8Quinolinyl Sulfonates in Aqueous Solution. Chem. Pharm. Bull. 2009, 57 (11), 1257-1266.

(24) Zeng, X.; Liu, S.; Shi, Z.; Xu, B., Hydrogen Bonding Cluster-Enabled Addition of Sulfonic Acids to Haloalkynes: Access to Both (E)- and (Z)Alkenyl Sulfonates. Org. Lett. 2016, 18 (19), 4770-4773.

(25) Pigulski, B.; Arendt, A.; Tomilin, D. N.; Sobenina, L. N.; Trofimov, B. A.; Szafert, S., Transition-Metal Free Mechanochemical Approach to Polyyne Substituted Pyrroles. J. Org. Chem. 2016, 81 (19), 9188-9198.

(26) Zhang, J.; Sun, J. Z.; Qin, A.; Tang, B. Z., Transition-Metal-Free Polymerization of Bromoalkynes and Phenols. Macromolecules 2019, 52 (8), 2949-2955.

(27) Niu, J.; Lunn, D. J.; Pusuluri, A.; Yoo, J. I.; O'Malley, M. A.; Mitragotri, S.; Soh, H. T.; Hawker, C. J., Engineering Live Cell Surfaces with Functional Polymers Via Cytocompatible Controlled Radical Polymerization. Nat. Chem. 2017, 9 (6), 537-545.

(28) Liu, Y.; Mao, L.; Liu, X.; Liu, M.; Xu, D.; Jiang, R.; Deng, F.; Li, Y.; Zhang, X.; Wei, Y., A Facile Strategy for Fabrication of Aggregation-Induced Emission (Aie) Active Fluorescent Polymeric Nanoparticles (Fpns) Via Post Modification of Synthetic Polymers and Their Cell Imaging. Mater. Sci. Eng., C 2017, 79, 590-595

(29) Wang, S.; Fu, C.; Wei, Y.; Tao, L., Facile One-Pot Synthesis of New Functional Polymers through Multicomponent Systems. Macromol. Chem. Phys. 2014, 215 (6), 486-492.

(30) Andraos, J.; Barclay, G. G.; Medeiros, D. R.; Baldovi, M. V.; Scaiano, J. C.; Sinta, R., Model Studies on the Photochemistry of Phenolic Sulfonate Photoacid Generators. Chem. Mater. 1998, 10 (6), 1694-1699.

(31) Iasilli, G.; Francischello, R.; Lova, P.; Silvano, S.; Surace, A.; Pesce, G.; Alloisio, M.; Patrini, M.; Shimizu, M.; Comoretto, D.; Pucci, A., Luminescent Solar Concentrators: Boosted Optical Efficiency by Polymer Dielectric Mirrors. Mater. Chem. Front. 2019, 3 (3), 429-436. (32) Battisti, A.; Ambrosetti, M.; Ruggeri, G.; Cappelli, C.; Pucci, A., A 4,4 -Bis(2-Benzoxazolyl)Stilbene Luminescent Probe: Assessment of Aggregate Formation through Photophysics Experiments and Quantum-Chemical Calculations. Phys. Chem. Chem. Phys. 2018, 20 (41), 26249-26258.

(33) Borelli, M.; Iasilli, G.; Minei, P.; Pucci, A., Fluorescent Polystyrene Films for the Detection of Volatile Organic Compounds Using the Twisted Intramolecular Charge Transfer Mechanism. Molecules 2017, $22(8), 1306$.

(34) Mark, J. E., Physical Properties of Polymers Handbook. Springer: 2007; Vol. 1076.

(35) Han, T.; Yao, Z.; Qiu, Z.; Zhao, Z.; Wu, K.; Wang, J.; Poon, A. W.; Lam, J. W. Y.; Tang, B. Z., Photoresponsive Spiro-Polymers Generated in Situ by C-H-Activated Polyspiroannulation. Nat. Commun. 2019, 10 (1), 5483.

(36) Yang, J.-C.; Ho, Y.-C.; Chan, Y.-H., Ultrabright Fluorescent Polymer Dots with Thermochromic Characteristics for Full-Color Security Marking. ACS Appl. Mat. Interfaces 2019, 11 (32), 29341-29349.

(37) Wang, Y.; Tang, Z.; Correa-Duarte, M. A.; Liz-Marzán, L. M.; Kotov, N. A., Multicolor Luminescence Patterning by Photoactivation of Semiconductor Nanoparticle Films. J. Am. Chem. Soc. 2003, 125 (10), $2830-2831$

(38) Devatha, G.; Rao, A.; Roy, S.; Pillai, P. P., Förster Resonance Energy Transfer Regulated Multicolor Photopatterning from Single Quantum Dot Nanohybrid Films. ACS Energy Lett. 2019, 4 (7), 1710-1716.

(39) Malak, S. T.; Jung, J.; Yoon, Y. J.; Smith, M. J.; Lin, C. H.; Lin, Z.; Tsukruk, V. V., Large-Area Multicolor Emissive Patterns of Quantum Dot-Polymer Films Via Targeted Recovery of Emission Signature. Adv. Opt. Mater. 2016, 4 (4), 608-619.

(40) Shen, H.; Wu, Y.; Fang, L.; Ye, S.; Wang, Z.; Liu, W.; Cheng, Z.; Zhang, J.; Wang, Z.; Yang, B., From 1d to 3d: A New Route to Fabricate Tridimensional Structures Via Photo-Generation of Silver Networks. RSC Advances 2015, 5 (36), 28633-28642.

(41) Wu, Y.; Zhang, K.; Yang, B., Ordered Hybrid Micro/Nanostructures and Their Optical Applications. Adv. Opt. Mater. 2019, 7 (7), 1800980. (42) Li, Y.; Zhang, J.; Liu, W.; Li, D.; Fang, L.; Sun, H.; Yang, B., Hierarchical Polymer Brush Nanoarrays: A Versatile Way to Prepare Multiscale Patterns of Proteins. ACS Appl. Mat. Interfaces 2013, 5 (6), 2126-2132. (43) Wu, P.; Wang, J.; Jiang, L., Bio-Inspired Photonic Crystal Patterns. Mater. Horiz. 2020, 7 (2), 338-365.

(44) Liu, L.; Wang, X.; Zhu, S.; Yao, C.; Ban, D.; Liu, R.; Li, L.; Wang, S., Controllable Targeted Accumulation of Fluorescent Conjugated 
Polymers on Bacteria Mediated by a Saccharide Bridge. Chem. Mater 2020, 32 (1), 438-447.

(45) Zhu, C.; Liu, L.; Yang, Q.; Lv, F.; Wang, S., Water-Soluble Conjugated Polymers for Imaging, Diagnosis, and Therapy. Chem. Rev. 2012, 112 (8) 4687-4735.

(46) Zhu, C.; Yang, Q.; Lv, F.; Liu, L.; Wang, S., Conjugated PolymerCoated Bacteria for Multimodal Intracellular and Extracellular Anticancer Activity. Adv. Mater. 2013, 25 (8), 1203-1208.

(47) Li, X.; Bai, H.; Yang, Y.; Yoon, J.; Wang, S.; Zhang, X., Supramolecular Antibacterial Materials for Combatting Antibiotic Resistance. Adv. Mater. 2019, 31 (5), 1805092.

(48) Li, P.; Poon, Y. F.; Li, W.; Zhu, H.-Y.; Yeap, S. H.; Cao, Y.; Qi, X.; Zhou, C.; Lamrani, M.; Beuerman, R. W.; Kang, E.-T.; Mu, Y.; Li, C. M.; Chang, M.
W.; Jan Leong, S. S.; Chan-Park, M. B., A Polycationic Antimicrobial and Biocompatible Hydrogel with Microbe Membrane Suctioning ability. Nat. Mater. 2011, 10 (2), 149-156.

(49) Luo, Y.; Wang, C.; Peng, P.; Hossain, M.; Jiang, T.; Fu, W.; Liao, Y.; Su, M., Visible Light Mediated Killing of Multidrug-Resistant Bacteria Using Photoacids. J. Mater. Chem. B 2013, 1 (7), 997-1001.

(50) Liu, X.; Li, M.; Han, T.; Cao, B.; Qiu, Z.; Li, Y.; Li, Q.; Hu, Y.; Liu, Z.; Lam, J. W. Y.; Hu, X.; Tang, B. Z., In Situ Generation of Azonia-Containing Polyelectrolytes for Luminescent Photopatterning and Superbug Killing. J. Am. Chem. Soc. 2019, 141 (28), 11259-11268. 